\title{
Stray capacitance of a two-layer air-cored inductor
}

\author{
M.J. Hole and L.C. Appel
}

\begin{abstract}
A new analytic method for predicting the stray capacitance of a double layer coil with conducting shield and air core is presented. The method involves construction of a two-layer turnto-turn circuit model, which includes the self-inductance and resistance of each turn, the mutual inductance representing the flux linkage between all turns, and the capacitance between adjacent turns and the capacitance between the outer turns and conducting shield. At high, low and zero frequency the model is reduced to an effective capacitance, inductance, and resistance respectively: for frequencies below the first self-resonant frequency the behaviour of the circuit is approximated by an RLC circuit. A new result is a recursive calculation of the stray capacitance of the high frequency circuit model. The method and stray capacitance value are compared to alternative models and stray capacitance predictions, and the limitations of the different approaches discussed. Comparisons with experimental measurements show good agreement at the fundamental selfresonance and confirm that the effect of a conducting shield is likely to be small for a closelywound coil.
\end{abstract}

\section{Introduction}

At high frequencies, the impedance of an inductor ceases to be ideal and is dominated by parasitic capacitances. The cumulative effect of these capacitances is commonly described as a 'stray' capacitance, $C_{s}$. Of particular interest is the frequency at which the capacitive and inductive impedances match, giving rise to a self-resonance, and providing an upper-frequency bound to operation as an inductor.

The magnitude of the stray capacitance is strongly dependent on the winding geometry and the proximity of any conducting surfaces. Calculation of it can be carried out directly, by solving numerically the electrostatic field equations [1], or by distributed capacitance modelling, in which the inductor is represented by a network of capacitance elements representing capacitances between discrete conducting elements [2, 3]. Although the former method is likely to be more accurate, particularly in the case of more complex geometries, the latter approach is particularly useful for scoping studies.

In the past, measurements of the stray capacitance have been inferred from low frequency measurements of the inductance, $L_{\text {coil }}$, coupled with measurements of the first self-resonant frequency, $f_{0}$ [2-4]. In instances where the inductance is also frequency dependent (e.g., inductors with a ferrite core), the addition of an external capacitor has been used to extract the inductance local to the self-resonant frequency [5].

\footnotetext{
(C) IEE, 2005

IEE Proceedings online no. 20045217

doi:10.1049/ip-cds:20045217

Paper first received 19th December 2004 and in final revised form 22nd July 2005

M.J. Hole is with the Research School of Physical Sciences and Engineering, Australian National University 0200, A.C.T. Australia

L.C. Appel is with EURATOM/UKAEA Fusion Association, Culham Science Centre, Abingdon, Oxon, OX14 3DB, UK

E-mail: mhole@physics.usyd.edu.au
}

In this paper, a method for modelling a double-wound coil with an air-core and conducting shield is presented. An array of these coils (OMAHA) has been installed into the Spherical Tokamak, MAST [6] for measuring magnetic fluctuations up to $5 \mathrm{MHz}$. Indeed, magnetic fluctuation activity, believed to be compressional Alfven eigenmodes at 3.5 MHz has already been detected using the OMAHA array [7]. The function of the OMAHA coils favours a double-wound winding configuration in order to maximise magnetic pick-up away from the coil, with a large turnsdensity to maximise the coil inductance per unit length, and whose first self-resonant frequency, $f_{0}$, is greater than $5 \mathrm{MHz}$. The principal objective of this article is to determine simple expressions for the stray capacitance. When combined with well-known expressions for the coil inductance, these expressions provide an estimate of $f_{0}$, and thus are a useful design tool. This work also complements a recent study by Appel and Hole [8], which provided a synopsis of coil in-situ remote calibration techniques of Heeter et al. [9].

The model developed here builds principally upon the work of Grandi et al. [2], in which a high frequency equivalent circuit was used to generate expressions for the stray capacitance of an $n$ turn single layer air-cored solenoid. In their work, a distributed capacitance network was used to determine an expression for the stray capacitance, valid at frequencies significantly above $f_{0}$, where the circuit model of the inductor retains only the effects of capacitive circuit components.

In this work, the single layer model is extended to provide predictions for the stray capacitance of a two-layer solenoid, thereby allowing comparison to the multiple layer distributed capacitance model of Massarini and Kazimierczuk [3]. Differences between the treatments are described in Section 3. As in Massarini and Kazimierczuk, generation of stray capacitance expressions for three or more layers is a straight-forward generalisation of the two-layer result. The advantage of distributed capacitance networks (e.g. Grandi et al., Massarini and Kazimierczuk, this work), as compared to full numerical solutions of the electrostatic field equations (e.g. Yu and Holmes [1]), lies in the model simplicity, which 
affords a simple and fast computation of the the stray capacitance. Measurements of the circuit model parameters have been obtained by a least squares fitting of the impedance profile over the frequency range $0<f<1.5 f_{0}$. To our knowledge, these are the first published results of both the predicted and measured stray capacitance of a double layer solenoid.

\section{Lumped-circuit model}

Figure $1 a$ is an illustration of the coil to be modelled. To describe the coil we introduce cylindrical co-ordinates $(r, \theta, z)$, with orientation as shown in Fig. 1a. The coordinate origin has been placed at the terminal end of the coil, and centred to the axis of the former. The nonconducting former has radius $R$ and length $l$, upon which are layered two closely packed helical windings. In this work the coil is taken to be sufficiently long such that the fringing of the magnetic and electric fields near the endpoints of the coil may be ignored. In addition, the different winding pitch (the axial direction of each winding) between inner and outer layers is ignored. Figure $1 b$ is an axial enlargement of four turns of Fig. 1a. As shown, the coil wire has a metal diameter of $2 a$, and is coated with a thin dielectric insulator of thickness $(d-2 a) / 2$, where $d$ is the total diameter of the wire. Finally, Fig. $1 c$ is a cross-section of the coil, labelling the inner and outer layers.

In general, the electrical properties of a coil are governed by both electrostatic and magnetostatic interactions, and a lumped-circuit model must include both. Figure $2 a$ is a lumped circuit representation of the $n$ turn coil, divided into turn-to-turn elements. Such a discrete circuit model will be valid providing the change in phase of a wave propagating from any turn is small along its length. That is, $(2 \pi R) / \lambda \ll 1$, with $\lambda$ the wavelength of excitation. The circuit model includes capacitive couplings between the $i$ th and $j$ th turns, the turn to shield coupling of the $i$ th outer turns, the self-inductance $L_{t, i}$ and resistance $R_{t, i}$ of each turn, and the mutual-inductance $M$ representing the fluxlinkage of each turn to all other $n-1$ turns. Only capacitive couplings between adjacent turns have been retained: this results from an extrapolation of the conclusions of Grandi et al., who found that for a single layer solenoid the neglect of capacitance between non-adjacent turns is reasonable providing that $|d /(2 a)|<2$. To simplify Fig. $2 a$, we next neglect differences between the turn-to-turn capacitance $C_{t t}$ of the inner and outer layers (i.e. same $z$, different $r$ ) and adjacent turns within the same layer (i.e. same $r$, different $z$ ). We also neglect differences between the capacitance of 'next' nearest neighbours, which are henceforth labelled $C_{t t}^{*}$. Finally, the variation in the turn-to-shield capacitance along the solenoid has been ignored. With these reductions, the circuit model of Fig. $2 a$ is reduced to Fig. 2b. In Section 3 we compute $C_{t t}^{*} / C_{t t}=0.1$ and $|d /(2 a)|=1.07$ for MAST coils, and so the neglect of capacitive coupling between more distant turns is not unreasonable.

In principal, analysis of the circuit shown in Fig. 2 will yield the full circuit response including the first self-resonant frequency $f_{0}$, and higher resonances owing to reflected waves coalescing with the same phase (i.e. transmission line effects). However, assuming the primary purpose is to establish $f_{0}$, a simpler approach is to model the coil characteristics in the two limits where the effect of the stray capacitance can be ignored (low frequency limit), and where the effect of the stray capacitance dominates (high frequency limit).

In the low frequency limit, Wheeler [10] developed a simple approximate formula for circular cross-section
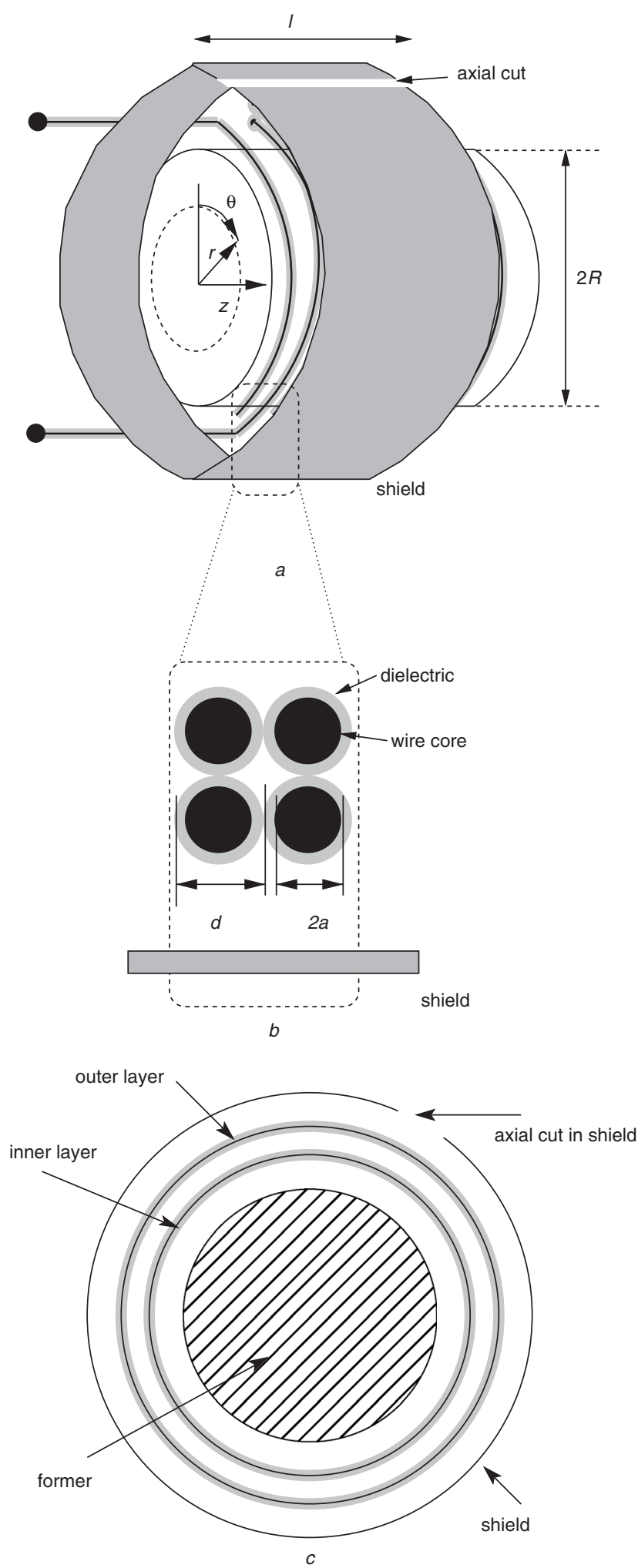

Fig. 1 Illustration of a densely packed two layer inductor $a$ Full geometry of the two layer windings and external shield $b$ Magnification of four turns of the inductor, with $a$ the wire radius and $d$ the diameter of the dielectric coated wire

$c$ Cross-section of the solenoid

inductors

$$
L_{\text {coil }}=\frac{\mu_{0} \pi n^{2} \bar{r}^{2}}{l+0.9 \bar{r}}
$$

correct to within $1 \%$ providing that $l /(2 r)>0.4$, where $\bar{r}$ is the mean coil radius. The coil resistance computes as $R_{\text {coil }}=n R_{t}(\omega)$, where $\omega=2 \pi f$ denotes a frequency 


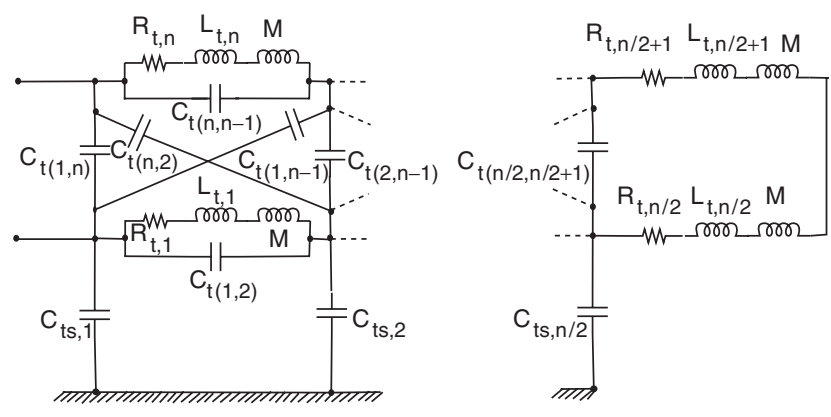

a
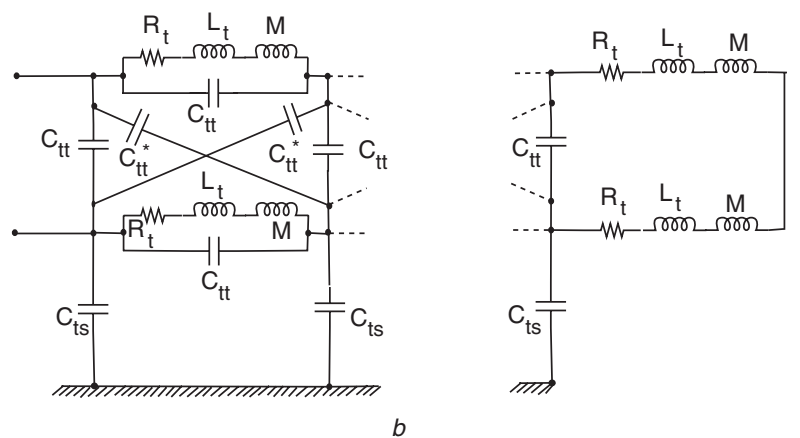

Fig. 2 Lumped-circuit representation of the coil

$a$ The labelled circuit components and couplings between the different turns

$b$ The simplified circuit, with the assumptions made in this work

dependence arising from the skin-effect. At the low frequency limit, $R_{t} \approx R_{D C, l}$, where

$$
R_{D C, l}=\frac{\rho l_{t}}{\pi a^{2}}
$$

and where $\rho$ is the conductor resistivity and $l_{t}$ the average length of one winding. A general expression for $R_{t}(\omega)$ is found from Bartoli [11]

$$
\begin{aligned}
R_{t}(\omega)= & R_{D C, l} \Re\left\{\frac{A(\omega)+i A(\omega)}{\tanh (A(\omega)+i A(\omega))}\right. \\
& \left.+\frac{2\left(N_{l}^{2}-1\right)}{3}(A(\omega)+i A(\omega)) \tanh (A(\omega)+i A(\omega))\right\}
\end{aligned}
$$

For a compactly wound coil with $d$ the spacing between coils, $A(\omega)$ can be written

$$
A(\omega)=\left(\frac{\pi}{4}\right)^{3 / 4} \frac{(2 a)^{3 / 2}}{\delta(\omega) d^{1 / 2}}
$$

with the skin depth $\delta(\omega)$ given by

$$
\delta(\omega)=\left(\frac{2 \pi \rho}{\mu_{r w} \mu_{0} \pi \omega}\right)^{1 / 2}
$$

where $\mu_{r w}$ is the conductor relative permeability.

At the high-frequency limit, the inductance limbs (i.e. $R_{t}$, $L_{t}, M$ paths) in Fig. 2 can be treated as an open circuit, enabling a simpler calculation of the stray-capacitance $C_{s}$. An estimate of the valid frequency range for this reduction is $\left|R_{t}+j \omega\left(L_{t}+M\right)\right| \gg\left|1 /\left(j \omega C_{t t}\right)\right|$, with $\omega=2 \pi f$ the angular frequency. That is, $f \gg f_{t}$ with

$$
\begin{aligned}
f_{t} & =1 /\left(2 \pi \sqrt{\left(L_{t}+M\right) C_{t t}}\right) \\
& =\sqrt{n} /\left(2 \pi \sqrt{L_{\text {coil }} C_{t t}}\right)
\end{aligned}
$$

where $L_{\text {coil }}=n\left(L_{t}+M\right)$, and the mutual inductance linking each turn with the other $n-1$ turns is
$M \approx(n-1) L_{t}$. In Section 3 we show a posteriori that this frequency is well above $f_{0}$. The coil characteristics over both frequency limits is represented approximately by an $R L C$ circuit, comprising the series combination of a resistor $R$ and coil inductance $L_{\text {coil }}$, in parallel with the stray capacitance $C_{s}$.

As in previous treatments [2,3], the effect of higher-order self-resonant phenomena on the value of stray capacitance, when used (together with the low frequency inductance) to predict the fundamental self-resonance, is neglected. Such effects are outside the scope of this work, which is to produce simple scoping design estimates for the stray capacitance. Qualitatively however, the presence of higher order resonances means that the measured fundamental self-resonance $f_{0}$ will be down-shifted from the predicted values computed in this work. To see this, we label the successive resonant frequencies in the circuit $f_{0}, f_{1}$, $f_{2}, \ldots, f_{N}$. In a frequency range local to each resonance, the circuit can be described by an $L C$ circuit, with $f_{i}=1 /\left(2 \pi \sqrt{ }\left(L_{i} C_{i}\right)\right)$. In general, the highest resonance of Fig. $2 b$ will be the self-resonance of the turn-to-turn element. For this element $L_{N} \approx L_{t}+M=L_{\text {coil }} / n$, with $L_{\text {coil }} \equiv L_{0}$. We combine $L_{0}$ and $C_{N}$ to yield a prediction for the fundamental self-resonant frequency, $f_{0, \text { pred }}=$ $1 /\left(2 \pi \sqrt{ }\left(L_{0} C_{N}\right)\right)$. As $f_{N}>f_{0}$ and $n \gg 1$, it follows that $C_{N}<C_{0}$ and $f_{0, p r e d}>f_{0}$. That is, the predicted frequency will be an upper estimate to the measured self-resonant frequency. More complete distributed circuit models, motivated by communications [12] and semiconductor [13] applications in the radio-frequency and microwave ranges, are able to be fitted to the measured impedance profile, but do not offer prescriptions for calculating the stray capacitance at the design stage. The development of such a predictive distributed model in realistic geometry would provide a more accurate design tool, able to resolve all the coil resonances.

\section{Calculation of stray-capacitance}

In the impedance domain, the capacitative coupling of any four turns (except those at the end) can be described by the circuit element shown in Fig. 3, where $Z_{\delta}=1 /\left(j \omega C_{t s}\right)$, $Z_{\gamma}=1 /\left(j \omega C_{t t}^{*}\right), Z_{\alpha}=Z_{\beta}=1 /\left(j \omega C_{t t}\right)$ and the current loops $I_{\alpha, i}, I_{\beta, i}, I_{\gamma, i}$, and $I_{\delta, i}$ are as drawn. For the final four turns, the capacitive coupling is described by the same circuit, except that $Z_{\beta}=0$ on the right hand side. Here, $j$ is complex notation, with $j^{2}=-1$. The issue of model completeness can be shown by current node analysis: only three current loops contribute to the output coil

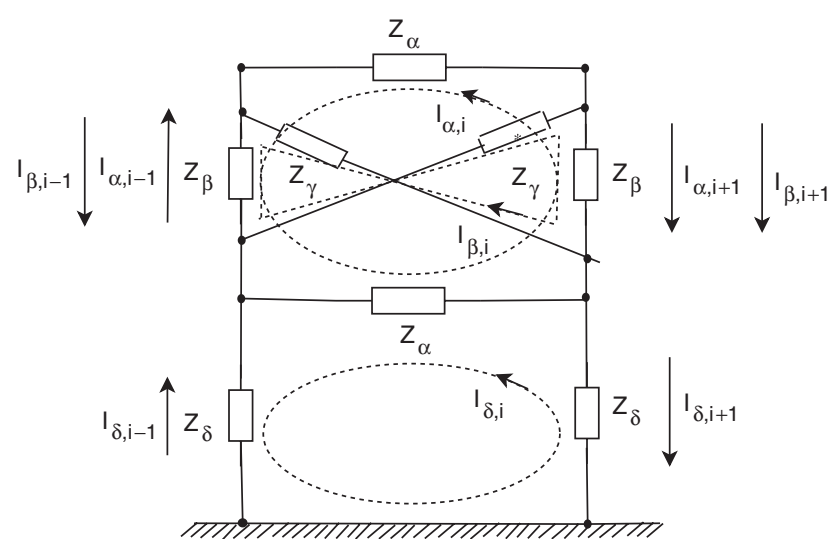

Fig. 3 One element of the lumped-circuit representation showing constituent current paths 
impedance: $I_{\delta, i}, I_{\beta, i}$, and $I_{\alpha, i}$. For all but the last circuit, Kirchhoff's voltage law may be written as the matrix equation

$$
\mathbf{Z} \mathbf{I}_{i}-\mathbf{L I}_{i-1}+\mathbf{R} \mathbf{I}_{i+1}=0
$$

where $\mathbf{I}_{i}$ is the column vector $\left(I_{\alpha, i}, I_{\beta, i}, I_{\delta, i}\right)$, and

$$
\begin{gathered}
\mathbf{Z}=\left(\begin{array}{ccc}
-Z_{\alpha} & 0 & 2 Z_{\delta}+Z_{\alpha} \\
0 & 2\left(Z_{\beta}+Z_{\gamma}\right) & 0 \\
2\left(Z_{\alpha}+Z_{\beta}\right) & 0 & -Z_{\alpha}
\end{array}\right) \\
\mathbf{L}=\left(\begin{array}{ccc}
0 & 0 & Z_{\delta} \\
Z_{\beta} & -Z_{\beta} & 0 \\
Z_{\beta} & -Z_{\beta} & 0
\end{array}\right) \\
\mathbf{R}=\left(\begin{array}{ccc}
0 & 0 & -Z_{\delta} \\
Z_{\beta} & Z_{\beta} & 0 \\
-Z_{\beta} & -Z_{\beta} & 0
\end{array}\right)
\end{gathered}
$$

External connections to the coil are taken to be left of the circuit at $i=1$. At the last element on the right hand side $\mathbf{I}_{p+1}=0$, with $I_{\beta, p}=I_{\delta, p}=0$. Current conservation implies $\mathbf{I}_{p}=\mathbf{C}_{p-1} \mathbf{I}_{p-1}$ with

$$
\mathbf{C}_{p-1}=\left(\begin{array}{ccc}
-1 & -1 & 0 \\
0 & 0 & 0 \\
0 & 0 & 0
\end{array}\right)
$$

For the second and third last elements,

$$
\begin{gathered}
\mathbf{I}_{p-1}=\left(\mathbf{Z}+\mathbf{R} \mathbf{Z}^{-1} \mathbf{L}\right)^{-1} \mathbf{L} \mathbf{I}_{p-2} \\
\mathbf{I}_{p-2}=\left(\mathbf{Z}+\mathbf{R}\left(\mathbf{Z}+\mathbf{R Z}^{-1} \mathbf{L}\right)^{-1} \mathbf{L}\right)^{-1} \mathbf{L}_{p-3}
\end{gathered}
$$

and so forth. This may be written more compactly as

$$
\mathbf{I}_{i}=\mathbf{C}_{i-1} \mathbf{I}_{i-1}
$$

with the $\mathbf{C s}$ generated by the recursion relation

$$
\mathbf{C}_{i-1}=\left(\mathbf{Z}+\mathbf{R C}_{i}\right)^{-1} \mathbf{L}, \quad i<p
$$

Solving recursively for $\mathbf{I}_{1}$ we calculate the output impedance

$$
Z_{\text {out }}=\left(\frac{I_{\alpha, 0}-I_{\beta, 0}-I_{\alpha, 1}-I_{\beta, 1}}{I_{\alpha, 0}-I_{\beta, 0}}\right) Z_{\beta}
$$

and extract the output stray capacitance, $C_{\text {out }}=1 /\left(j \omega Z_{\text {out }}\right)$.

Expressions for the output stray capacitance have been checked in a number of physically relevant special cases. The stray capacitance of a single layer solenoid without shielding can be obtained by decoupling the inner and outer layers. This involves setting $Z_{\gamma} \rightarrow \infty, Z_{\delta} \rightarrow \infty$, and $Z_{\beta} \rightarrow$ $\infty$ of all but the last circuit, yielding $C_{s}=C_{t t} /(n-1)$, which is consistent with the results of Grandi et al. for a single layer unshielded solenoid. If the turn-to-turn capacitance within layers dominates over the turn-to-turn capacitance between layers (i.e. $Z_{\alpha} / Z_{\beta} \rightarrow 0$, then $C_{s} \rightarrow(n / 2) C_{t t}+(n-2) C_{t t}^{*}$. In the reverse ordering, where the turn-to-turn capacitance between layers dominates the turn-to-turn capacitance within layers (i.e. $Z_{\beta} / Z_{\alpha} \rightarrow 0$ ), $C_{s} \rightarrow C_{t t}$.

Figure $4 a$ plots the normalised stray capacitance against the normalised diagonal turn-to-turn capacitance for $n=4,6,8$ and 10 , with the shield at infinity $\left(C_{t s} \rightarrow 0\right)$. The swift convergence with increasing $n$ results from the series and diagonal turn-to-turn capacitance, which successively reduce the contribution from higher turns. Figure $4 b$ plots the normalised stray capacitance against the normalised turn-to-shield capacitance for $n=4,6,8$ and 10, and $C_{t t}^{*} / C_{t t}=0.1$ (MAST case). As in Fig. $4 a$, the convergence with increasing $n$ results from the series and diagonal turnto-turn capacitance. In the limit of large $C_{t s}$ the series turnto-turn capacitance is short circuited, and it is the diagonal

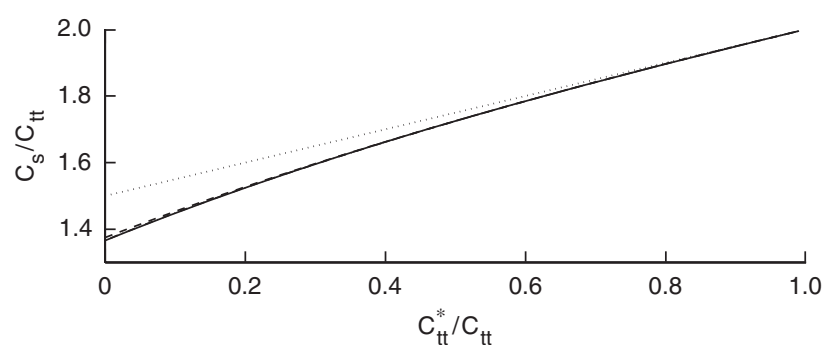

a

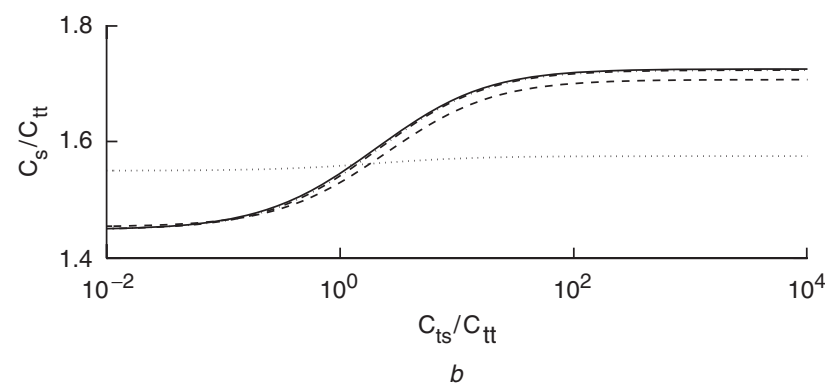

Fig. 4 Plot of the normalised stray capacitance $C_{s} / C_{t t}$ against $C_{t I}^{*} /$ $C_{t t}$ and $C_{t s} / C_{t t}$

$a$ Normalised diagonal turn-to-turn capacitance $C_{t t}{ }^{*} / C_{t t}$ with no shield $C_{t s}=0$

$b$ Normalised turn-to-shield capacitance $C_{t s} / C_{t t}$ with $C_{t t}^{*} / C_{t t}=0.1$ The different lines are: $n=4$ (dots), $n=6$ (dashes), $n=8$ (dashdot), and $n=10$ (solid)

turn-to-turn capacitance that couples the shield and higher order circuits. Figure $4 b$ shows that the maximal effect of a shield is to increase the stray capacitance by around $20 \%$. However, this requires $C_{t s} / C_{t t} \geq 10$, which can only be achieved by a very closely fitting shield; for a shield placed more than distance $d$ from the coil, $C_{t s} / C_{t t} \lesssim 1$, and the increase in stray capacitance will be $<7 \%$.

In the two extremes that a shield is absent and the shield is adjacent to the inductor, the stray capacitance reduces to $C_{s}=1.45 C_{t t}$ and $C_{s}=1.72 C_{t t}$, respectively, where $n \geq 10$ and $C_{t t}^{*} / C_{t t}=0.1$. These results compare well to earlier calculations by Massarini and Kazimierczuk, who found that for a two layer winding with $n \geq 10$, the stray capacitance reduces to $C_{s}=1.62 C_{t t}$ for no shield, and $C_{s}$ $=1.83 C_{t t}$ for a conducting core. Differences between the expressions occur because in this model the windings lie in cylindrical shells centred at radius $r=R$ and $r=R+d$, as opposed to the completely stacked layers of Massarini and Kazimierczuk, which lie in cylindrical shells of radius $r=R$ and $r=R+2 d / \sqrt{ } 3$. Both treatments have error associated with windings of opposite winding pitch. Turns of opposite pitch do not lie compactly stacked, and so the spacing between layers will be underestimated in computations of $C_{t t}$ in Massarini and Kazimierczuk. Whilst the spacing between layers is correct in this treatment, the fact that the winding pitch is opposite between layers is neglected. Thus, the stray capacitance, parameterised by the turn-to-turn capacitance of touching wires, will be an overestimate, as turns in different layers will in general be non-touching. In turn, the predicted self-resonant frequency will be an underestimate.

Finally, in order to compute the stray capacitance, expressions for $C_{t t}$ and $C_{t t}^{*}$ must be determined. Formal solutions require calculation of the electrostatic field within the windings. As mentioned, this is outside the scope of this work, which is designed to provide simple scoping formulas. Instead, we have used expressions for the capacitance in 
various limiting cases. For $C_{t t}$, the conductors are assumed to be immersed in a medium of permittivity $\varepsilon_{d}$ [14]

$$
C_{t t}=\frac{\pi l_{t} \varepsilon_{d}}{\cosh ^{-1}\left(\frac{d}{2 a}\right)}
$$

Here, $l_{t}$ is the length of wire in one turn. For media in which $\varepsilon_{d} \gg \varepsilon_{a i r}$, the permittivity of air, (18) will significantly overestimate $C_{t t}$, as the electric field lines between the windings will be different to those in a homogeneous medium. Even when $\varepsilon_{d} \approx \varepsilon_{a i r}$ however, (18) will slightly overestimate $C_{t t}$, as all electric field lines do not start and end on adjacent conductors. That is, the presence of other conductors is not taken into account. Consequently, (18) should be taken as an upper bound to $C_{t t}$, and therefore a lower bound to $f_{0}$. For $C_{t t}^{*}$, we have used the equivalent capacitance of the series combination of the capacitance of the insulated coating and the capacitance related to the air gap between the turns [2]. This yields

$$
C_{t t}^{*}=\frac{\pi l_{t} \varepsilon_{\text {air }}}{\ln \left(F+\sqrt{F^{2}-(1+t / a)^{2 \varepsilon_{\text {air }} / \varepsilon_{d}}}\right)}
$$

where $t$ is the thickness of the dielectric coating and

$$
F=\frac{d / \sqrt{2} a}{(1+t / a)^{1-\varepsilon_{a i r} / \varepsilon_{d}}}
$$

Taken together, (18) and (19), and Fig. 4 provide an approximate description for determining the stray capacitance of the circuit in the absence and presence of shielding.

Finally, for completeness, we note that the self-resonant frequency of the $R L C$ circuit is

$$
\begin{aligned}
f_{0} & =1 /\left(2 \pi \sqrt{L_{\text {coil }} C_{s}}\right) \approx 1 /\left(2 \pi \sqrt{L_{\text {coil }} C_{t t}}\right) \\
& =f_{t} / \sqrt{n}
\end{aligned}
$$

and so $f_{0} \ll f_{t}$ providing $n \gg 1$. In turn, reduction of the circuit model of Fig. $2 a$ to Fig. $2 b$ with open circuit inductance limbs, for the purposes of calculating $C_{s}$ at frequencies above $f_{0}$ was reasonable.

The expressions used in this work for $C_{t t}$ and $C_{t t}^{*}$ differ to earlier treatments in several respects. In Massarini and Kazimierczuk, the electric field path in an infinite compact lattice was assumed, and expressions for the capacitance per unit surface area between connecting field lines derived. The turn-to-turn capacitance was found by integrating over all possible paths. In Grandi, where a single layer solenoid was modelled, (19) was used for $C_{t t}^{*}$, and (18) with $\varepsilon_{d} \rightarrow \varepsilon_{\text {air }}$ used to describe $C_{t t}$ for windings with large air spacing between turns. For the double layer, oppositely wound coil considered here, an infinite fixed lattice structure cannot be assumed: turn-to-turn capacitance calculation requires a full 3-D Maxwell electrostatic simulation (e.g. [1]). Given the application of this model is to provide simple scoping formulas, we have instead followed the simplified approach of Grandi.

\section{Experimental validation}

To test the stray capacitance model, the characteristics of a candidate double-layer coil to be used in MAST were measured. The specifications of the coil were $n=30$, $l=14 \mathrm{~mm}, a=0.38 \mathrm{~mm}, d=0.81 \mathrm{~mm}$, with the coil wire coated in a $t=25 \mu \mathrm{m}$ layer of polyamide/imide insulator with relative permittivity $3.9 \leq \varepsilon_{d} / \varepsilon_{0} \leq 5.4$ [15]. The outer diameter of the wound coil was $23 \mathrm{~mm}$, giving a mean turns radius, $\bar{r}$, of $\bar{r}=10.7 \mathrm{~mm}$, and coil length to radius ratio $l / \bar{r}=1.3$, which is within the range of applicability for (1) (however since the formula is intended for a single-layer winding, application to a double-layer solenoid may result in an error which is more than $1 \%$ ). From (1), (2), (18), and (19), the derived parameters are $\quad L_{\text {coil }}=17.2 \mu \mathrm{H}, \quad R_{D C} \equiv n R_{D C, l}=67.5 \mathrm{~m} \Omega, \quad$ and $20.2 \mathrm{pF} \leq C_{t t} \leq 28.0 \mathrm{pF}$ with $C_{t t}^{*}=2.1 \mathrm{pF}$. Using Fig. 4 to obtain the ratio $C_{s} / C_{t t}$ for $n \geq 10$, one obtains $29.3 \mathrm{pF} \leq C_{s} \leq 40.6 \mathrm{pF}$, with mean $\bar{C}_{s}=35.0 \mathrm{pF}$ (overbar denotes mean). The large uncertainty in the predicted stray capacitance $( \pm 16 \%)$ corresponds to the uncertainty in the permittivity of the insulator.

The impedance profile of the coil was measured with a ZVRE Rhodes and Schwarz $3 \mathrm{GHz}$ network analyser [16]. Measurements of the impedance, $Z_{\text {meas }}$, were obtained from the $S_{11}$ reflection coefficient [17]. Figure 5 shows the absolute value of the impedance, $|Z|$, over a $200 \mathrm{kHz}-$ $15 \mathrm{MHz}$ sweep range. Whilst the fundamental resonance $f_{0}$ is defined by $\mathfrak{J}\{Z\}=0$, for practical reasons, the fundamental resonance was measured to be the frequency at which $\Re\left\{Z_{\text {meas }}\right\}$ first peaked. The validity of this assumption can be examined by noting the predicted impedance $[11,18]$ can be written

$$
Z_{\text {pred }}=\frac{R_{\text {coil }}+j \omega L_{\text {coil }}\left(1-\omega^{2} L_{\text {coil }} C_{s}-C_{s} R_{\text {coil }}^{2} / L_{\text {coil }}\right)}{\left(1-\omega^{2} L_{\text {coil }} C_{s}\right)^{2}+\omega^{2} C_{s}^{2} R_{\text {coil }}^{2}}
$$

If $C_{s} R_{\text {coil }}^{2} / L_{\text {coil }} \ll 1$ then at $\omega=2 \pi f_{0}=1 / \sqrt{ }\left(L_{\text {coil }} C_{s}\right)$, one finds $\mathfrak{\Im}\left\{Z_{\text {pred }}\right\}=0$ and $\partial \Re\left\{Z_{\text {pred }}\right\} / \partial \omega=0$. Hence, providing that the measured impedance profile can be closely fitted by (22) and $C_{s} R_{\text {coil }}^{2} / L_{\text {coil }} \ll 1$ (proven $a$ posteriori), peaks of $\Re\left\{Z_{\text {meas }}\right\}$ lie at the fundamental resonance $f_{0}$. Applying this technique to 10 measurements yields $f_{0}=5.32 \pm 0.02 \mathrm{MHz}$, where the error of $0.02 \mathrm{MHz}$ is inferred from the variance of the scatter of ten $f_{0}$ measurements. Higher order self-resonant frequencies, discussed elsewhere (e.g. Rhea [12]), can also be identified, at $30 \mathrm{MHz}$ and $74 \mathrm{MHz}$.

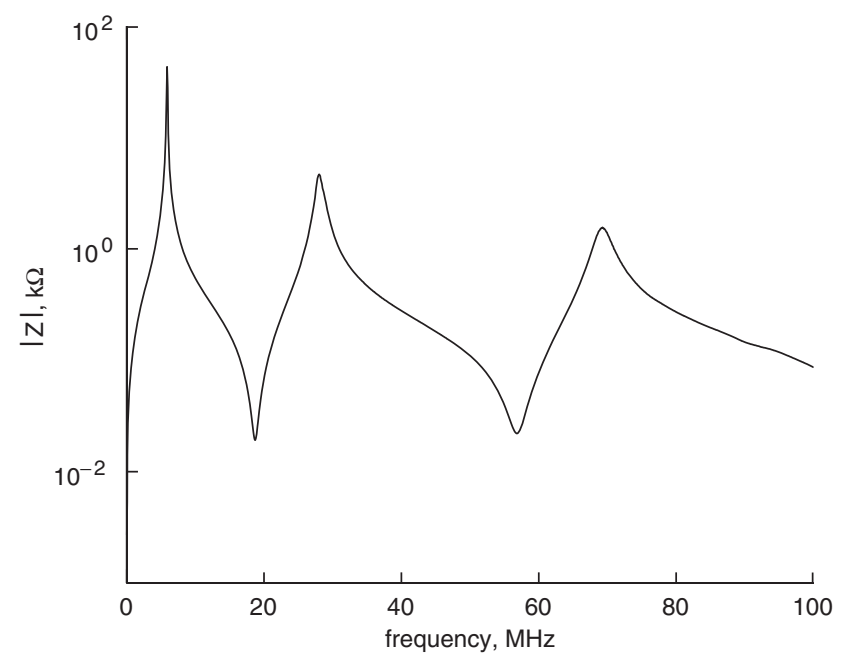

Fig. 5 Frequency dependence of the absolute value of the coil impedance, $|Z|$, as measured by a ZVRE Rhodes and Schwarz network analyser

Figures $6 a$ and $b$ show an enlargement of the magnitude of the imaginary part of $Z,|\Im\{Z\}|$, about $f_{0}$. The frequency response below $f_{0}$ is dominated by the coil inductance, and immediately above it by the coil capacitance. To obtain 

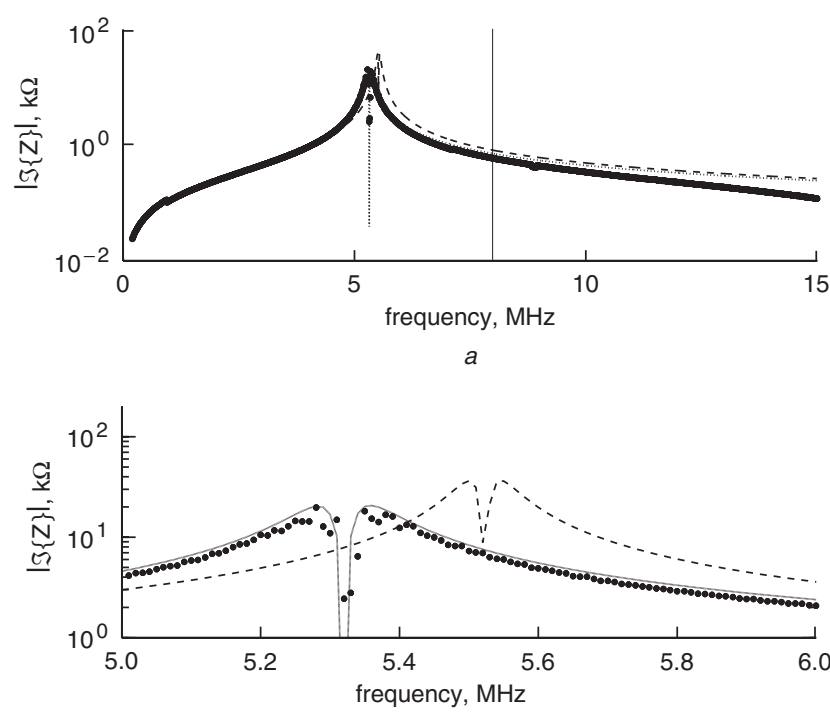

b

Fig. 6 Measured, fitted and predicted magnitude of the imaginary part of $Z,|\mathfrak{I}\{Z\}|$, about the fundamental resonant frequency, $f_{0}$

Solid points are the measured data, the dotted and dashed lines are the fitted and predicted profiles, respectively. At frequencies lower than $f_{0}=5.32 \pm 0.02 \mathrm{MHz}$, the coil is inductive (i.e. $\mathfrak{I}\{Z\}>0$ ), and immediately above it, the coil is capacitative (i.e. $\mathfrak{J}\{Z\}<0$ )

$a$ Vertical line denotes the upper frequency limit used in fitting to an $R L C$ parallel circuit

$b$ Enlargement of Fig. $6 a$ about the self-resonant frequency

fitted values for the $R L C$ circuit model parameters (i.e. frequency-independent measured values of $R_{D C}, L_{c o i l}$, and $C_{s}$ ), a least squares fit to the residue

$$
\begin{aligned}
r_{\text {fit }}(f)= & \mid\left(Z_{\text {pred }}(f)-Z_{\text {meas }}(f)\right) /\left(Z_{\text {pred }}(f)\right. \\
& \left.+Z_{\text {meas }}(f)\right) \mid
\end{aligned}
$$

was performed, with $Z_{\text {pred }}$ given by (22). The frequency range has been restricted to $0<f<1.5 f_{0}$, so as to avoid phenomena associated with the second self-resonant frequency. In each fit, the components $R_{D C}, L_{\text {coil }}$ and $C_{S}$ were varied to minimise the integral $L_{f i t}=\left(\int_{0}^{1.5 f_{0}} r_{f i t}(f) d f\right) /$ $\left(1.5 f_{0}\right)$ using a simplex search algorithm [19] implemented in Matlab. Initial values were taken to be the predicted values, whilst the exit condition was $\Delta I_{\text {fit }}<10^{-8}$ with $\Delta I_{f i t}$ the change in $I_{f i t}$ between iterations. For this fractional exit condition, $I_{f i t}<1.8 \times 10^{-2}$ for all experiments. Least squares fitting over 10 experiments yields: $R_{D C}=$ $115 \pm 3 \mathrm{~m} \Omega, L_{\text {coil }}=17.29 \pm 0.01 \mu \mathrm{H}, C_{s}=51.75 \pm 0.25 \mathrm{pF}$, where the variance in $R_{D C}, L_{c o i l}$ and $C_{S}$ has been taken from the scatter of the fits. The good agreement between the fitted (dotted line) to the measured (solid points) impedance profiles in Figs. $6 a$ and $b$ provides confidence in the circuit model. For completeness, we note that at $\bar{f}_{0}, R_{\text {coil }}=4.7 \Omega$, yielding $C_{S} R_{\text {coil }}^{2} / L_{\text {coil }} \approx 7 \times 10^{-5} \ll 1$, and so the fundamental resonance $f_{0}=1 /\left(2 \pi \sqrt{ }\left(L_{\text {coil }} C_{s}\right)\right)$ corresponds to $\partial \Re\left\{Z_{\text {pred }}\right\} / \partial \omega=0$ in (22).

To compare the fitted capacitance to the predicted capacitance, the value of $R_{D C}$ and $C_{S}$ must be reduced by $R_{D C \text {,test }}=31.1 \mathrm{~m} \Omega$ and $C_{\text {test }}=13.78 \mathrm{pF}$, being the d.c. resistance and capacitance of the connecting test board and cable measured (by a similar fitting technique) in isolation. This yields $\quad R_{D C, \text { fit }}=84 \pm 3 \mathrm{~m} \Omega, \quad L_{\text {coil }, \text { fit }}=17.29 \pm$ $0.01 \mu \mathrm{H}, C_{s, \text { fit }}=37.97 \pm 0.25 \mathrm{pF}$, values which compare well to the predicted values $R_{D C \text {, pred }}=67.5 \mathrm{~m} \Omega, L_{\text {coil, pred }}=$ $17.2 \mu \mathrm{H}, C_{s, \text { pred }}=35.0 \pm 5.7 \mathrm{pF}$. The discrepancy between $R_{D C}$ values may be the result of additional conducting structures in the test board, which cannot be adequately represented by the cylindrical wire model of (3)-(5). The predicted coil inductance is accurate to within $1 \%$ of the fitted value, consistent with the estimates of accuracy of (1) as proposed by Wheeler [10]. The fitted capacitance lies within the range of the predicted values, with mean $\bar{C}_{s, \text { pred }}=35.0 \mathrm{pF}, 8 \%$ below the fitted value. A more detailed comparison between the predicted and measured stray capacitance would require the permittivity of the insulator to be measured. Finally, the predicted inductance, $L_{\text {coil, pred }}=17.2 \mu \mathrm{H}$, and stray capacitance $C_{s, \text { pred }}=35.0 \pm 5.7 \mathrm{pF}$ combine to yield the prediction $f_{0}=6.5 \pm 0.5 \mathrm{MHz}$. If the capacitance of the test board and cable is added in parallel, then $1 / 2 \pi \sqrt{ }\left(L_{\text {coil }}\left(C_{s}+C_{\text {test }}\right)\right)=$ $5.5 \pm 0.3 \mathrm{MHz}$. As with $C_{s}$, the measured value for $f_{0}=$ $5.32 \pm 0.02 \mathrm{MHz}$ lies within the predicted range. The predicted impedance profile, correcting for the test board, is also shown in Figs. $6 a$ and $b$.

It is useful to compare these predictions to those of Massarini and Kazimierczuk [3]. Using their expression for a two layer stacked coil structure in Massarini and Kazimierczuk [3], $C_{s}=1.62 C_{t t}$, together with (15) for $C_{t t}$ in that article, yields $17.7 \mathrm{pF} \leq C_{s} \leq 21.5 \mathrm{pF}$, with mean $19.6 \mathrm{pF}$. The mean represents a $-46 \%$ error from the measured value, thereby casting doubt on the accuracy of the calculations of Massarini and Kazimierczuk [3] in predicting the stray capacitance on double wound inductors. It should be noted that the experimental measurements of Massarini and Kazimierczuk [3], which quoted an error of $-17.2 \%$ for the predicted stray capacitance compared to the measured value, were based only on a single layer inductor. The difference between the results of Massarini and Kazimierczuk [3] and our working stems from a difference in $C_{S} / C_{t t}\left(1.62\right.$ against 1.45) and $\bar{C}_{t t}$, $\left(\bar{C}_{t t}=24.1 \mathrm{pF}\right.$ against $\left.\bar{C}_{t t}=12.1 \mathrm{pF}\right)$ respectively. Physically, the value of $\bar{C}_{t t}$ in Hole and Appel is higher because the whole structure is assumed to be completely immersed in a dielectric.

Table 1 shows calculated values for $C_{t t}, C_{t t}^{*}$ and $C_{s}$ for different models, and over the range of permittivity of the polyamide/imide (PAI) insulator, $3.9 \leq \varepsilon_{r} \leq 5.4$. For completeness, we also show predictions for Hole and Appel, and Massarini and Kazimierczuk [3] in the limit that the entire structure is immersed in a dielectric (with permittivity $\varepsilon_{a i r}=\varepsilon_{r} \varepsilon_{0}$ ), where $\varepsilon_{0}$ is the permittivity of free space. In these cases, the predictions of the two models overlap more closely: differences are due to details of the electric field path, which have not been calculated precisely

Table 1: Predictions for $C_{t t}, C_{t t}^{*}$ and $C_{s}$ across different models and permittivity

\begin{tabular}{llllll}
\hline & $\varepsilon_{\text {air }}$ & $\varepsilon_{r}$ & $C_{t t}$ & $C_{t t}^{*}, \theta^{*}$ & $C_{s}$ \\
\hline Hole and Appel & $\varepsilon_{0}$ & 3.9 & $20.2 \mathrm{pF}$ & $2.1 \mathrm{pF},-$ & $29.3 \mathrm{pF}$ \\
Hole and Appel & $\varepsilon_{0}$ & 5.4 & $28.0 \mathrm{pF}$ & $2.1 \mathrm{pF},-$ & $40.0 \mathrm{pF}$ \\
Hole and Appel & $3.9 \varepsilon_{0}$ & 3.9 & $20.2 \mathrm{pF}$ & $7.7 \mathrm{pF},-$ & $33.5 \mathrm{pF}$ \\
Hole and Appel & $5.4 \varepsilon_{0}$ & 5.4 & $28.0 \mathrm{pF}$ & $10.7 \mathrm{pF},-$ & $46.2 \mathrm{pF}$ \\
$\begin{array}{l}\text { Massarini and } \\
\text { Kazimierczuk }\end{array}$ & $\varepsilon_{0}$ & 3.9 & $10.9 \mathrm{pF}$ &,$- 0.18 \mathrm{rad}$ & $17.7 \mathrm{pF}$ \\
$\begin{array}{l}\text { Massarini and } \\
\text { Kazimierczuk }\end{array}$ & $\varepsilon_{0}$ & 5.4 & $13.3 \mathrm{pF}$ &,$- 0.15 \mathrm{rad}$ & $21.5 \mathrm{pF}$ \\
$\begin{array}{l}\text { Massarini and } \\
\text { Kazimierczuk }\end{array}$ & $3.9 \varepsilon_{0}$ & 3.9 & $20.7 \mathrm{pF}$ &,$- 0.36 \mathrm{rad}$ & $27.9 \mathrm{pF}$ \\
$\begin{array}{l}\text { Massarini and } \\
\text { Kazimierczuk }\end{array}$ & $5.4 \varepsilon_{0}$ & 5.4 & $23.8 \mathrm{pF}$ &,$- 0.36 \mathrm{rad}$ & $38.6 \mathrm{pF}$ \\
\hline
\end{tabular}


in either structure. The similarity in predictions of $C_{s}$ between Massarini and Kazimierczuk with $\varepsilon_{a i r}=\varepsilon_{r} \varepsilon_{0}$ and Hole and Appel with $\varepsilon_{\text {air }}=\varepsilon_{0}$ occurs because in both cases $C_{t t}$ (which in Hole and Appel is the dominant capacitance) is calculated assuming immersion of the structure in the dielectric.

Finally, the effect of a shield was studied by measuring the variation in the frequency of the fundamental resonance. In these experiments, two thin copper sleeves of axial length $8 \mathrm{~mm}$ and $11 \mathrm{~mm}$, and with an axial cut, were wrapped around the terminal end of the coil, and molded to minimise the air gap (see Fig. 1 schematic). The axial cut ensured that currents in the shield did not affect the axial magnetic field, and thereby the coil inductance. The effects of conducting shields on the coil inductance have been examined elsewhere [20]. In the shield experiments described here, the measured fundamental resonance changed to $f_{0}=5.37 \pm 0.02 \mathrm{MHz}$ and $f_{0}=5.36 \pm 0.04 \mathrm{MHz}$ for the wide and narrow shields, respectively.

Correcting for the capacitance of the test board and cable, corresponding fits to $R_{D C}, L_{\text {coil }}$ and $C_{S}$ yielded $R_{D C}=75.8 \pm 1.9 \mathrm{~m} \Omega, L_{\text {coil }}=17.28 \pm 0.02 \mu \mathrm{H}, C_{s}=37.00 \pm$ $0.36 \mathrm{pF}, \quad$ and $\quad R_{D C}=76.2 \pm 0.1 \mathrm{~m} \Omega, \quad L_{\text {coil }}=17.17 \pm$ $0.02 \mu \mathrm{H}, C_{s}=37.69 \pm 0.65 \mathrm{pF}$, for the wide and narrow shield, respectively. In both cases, the experimentally derived values of $C_{s}$ are equal to the unshielded stray capacitance, to within error bounds; whilst the difference in self-resonant frequencies between the shielded and unshielded cases is attributable to the change in coil inductance. A possible reason why the stray capacitance has not changed is that each turn is almost-touching the shield, which is locally flat. Hence, we would expect $C_{t s} / C_{t t} \approx 1$, and so $C_{s} / C_{t t} \approx 1.55$ (see Fig. $6 b$ ), yielding $31.3 \leq C_{s} \leq 43.4 \mathrm{pF}$, with mean $\bar{C}_{s}=37.4 \mathrm{pF}$, which is $2.4 \mathrm{pF}$ above from the unshielded prediction of $35.0 \mathrm{pF}$. Whilst the difference exceeds the uncertainty in the measurements, the uncertainties in $C_{t s} / C_{t t}$ preclude a definitive comparison of theory to measurements. A series of more dedicated experiments, possibly involving the use of a conducting paint would be required to fully explore the upper limit of $C_{s} / C_{t t}=1.7$, shown in Fig. $4 b$.

\section{Conclusions}

A simple distributed circuit model has been developed to compute the stray capacitance, $C_{s}$, of a double layer aircored solenoid. The use of recursive circuit modelling techniques provide a prescription for generating algebraic expressions for the stray capacitance of an arbitrary number of coil turns. Providing that the number of coil turns is larger than 10, our analysis shows that the stray capacitance is proportional to the turn-to-turn capacitance of two wires immersed in a dielectric of permittivity $\varepsilon_{d}$ : for no shield, $C_{s}=1.45 C_{t t}$, with a shield, $C_{s} \leq 1.7 C_{t t}$. Qualitatively, these results consolidate earlier findings $[2,3]$ that the stray capacitance is only weakly dependant on the number of turns, and converges quickly with increasing number of turns, $n$. In a single-layer solenoid [2] it is the capacitive coupling with the shield that dominates the stray capacitance, and is responsible for the swift convergence with increasing $n$. In a two layer solenoid swift convergence with $n$ is achieved without the shield, and is due to the capacitive coupling between the first few turns in the inner and outer layer. We hypothesise that this result will extend to solenoids with an even number of layers, where the terminals are at the same end of the coil. In solenoids with an odd number of layers, the capacitance will in general be dominated by the electrical path through the shield.
The practical use of these estimates is that, when combined with existing expressions for the coil inductance, $L_{\text {coil }}$, an estimate of the fundamental self-resonant frequency of the coil, $f_{0}$, can be obtained. Because our model: (1) assumes that touching turns are immersed in a dielectric of permittivity $\varepsilon_{d}>\varepsilon_{0}$, and (2) does not account for the opposite winding pitch of the layers, the predicted values of $C_{s}$ and $f_{0}$ are likely to be upper and lower limits, respectively. Nonetheless, the expressions do provide a useful scoping tool to determine the maximum frequency operation as an inductor.

Experimental results were presented for a coil designed to measure high frequency oscillatory magnetic fields in a fusion plasma. The measured value of the stray capacitance was determined by a least squares fit to an $R L C$ circuit model over the frequency range $0 \leq f \leq 1.5 f_{0}$. Our results show that the measured stray capacitance is within the range of the predicted stray capacitance: uncertainties in the permittivity of the insulator give a range of $\pm 16 \%$, with the mean value of the stray capacitance $\bar{C}_{s}=35.0 \mathrm{pF}$, which is $8 \%$ lower than the measured value. A more detailed comparison between the predicted and measured stray capacitance would require the permittivity of the insulator to be measured. When combined with an expression for the coil inductance, the predicted selfresonant frequency $f_{0}$ is within the range of the measured value, with mean $4 \%$ larger than the measured value. Our results compare well to predictions of other models. For example, the analysis of Massarini and Kazimierczuk [3] predicts a stray capacitance and self-resonant frequency $46 \%$ below and $25 \%$ above the measured values, respectively. In both treatments, the higher mean of the predicted self-resonant frequency is also consistent with a frequency upshift owing to the presence of higher-order resonances discussed in Section 2.

Finally, the effect of a shield on the value of $C_{s}$ is predicted to be insignificant for the usual case of closelyspaced windings when $C_{t s} / C_{t t}<0.1$. In the case of widely spaced windings where $C_{t s} / C_{t t}>10, C_{s}$ is predicted to increase by not more than $20 \%$. Experimental measurements using the test coil confirm that a close fitting shield (with axial cut) has a negligible effect on the stray capacitance.

New results in this work are:

1. The construction of a simple model for the stray capacitance of a two-layer, tightly packed inductor. The model builds upon existing expressions for the turnto-turn capacitance, but differs to earlier multi-layer models because the coils are not assumed to be compactly packed.

2. Predictions for the unshielded stray capacitance and selfresonant frequency, which overlap the measured range. To our knowledge, these are the first published results of both the predicted and measured stray capacitance of a double layer solenoid. More precise predictions would require measurement of the permittivity of the insulator.

3. Predictions, validated by experiment, that the effect of a close-fitting conducting shield on the value of $C_{s}$ and $f_{0}$ is weak.

\section{Acknowledgments}

This work was funded jointly by the United Kingdom Engineering and Physical Sciences Research Council and by EURATOM. The authors would like to thank R. Martin for providing a test coil, and the referees for useful feedback and advice. 


\section{References}

$1 \mathrm{Yu}, \mathrm{Q}$., and Holmes, T.W.: 'A study on stray capacitance modeling of inductors by using the finite element method', IEEE Trans. Electromagn. Compat., 2001, 143, (1), pp. 88-93

2 Grandi, G., Kazimierczuk, M.K., Massarini, A., and Reggiani, U.: 'Stray capacitances of single-layer solenoid air-core inductors', IEEE Trans. Ind. Appl., 1999, 35, (5), pp. 1162-1168

3 Massarini, A., and Kazimierczuk, M.K.: 'Self-capacitance of inductors', IEEE Trans. Power Electron., 1997, 12, (4), pp. 671-676

4 Bartoli, M., Noferi, N., Reatti, A., and Kazimierczuk, M.K.: 'Modeling winding losses in high-frequency power inductors', J. Circuits Syst. Compat., 1995, 5, (4), pp. 607-626

5 Yu, Q., Holmes, T.W., and Naishadham, K.: 'RF equivalent circuit modeling of ferrite-core inductors and characterization of core materials', IEEE Trans. Electromagn. Compat., 2002, 44, (1), pp. $258-262$

6 Hole, M.J., Akers, R.J., Appel, L.C., Buttery, R.J., Brickley, C., Conway, N.J., Gryaznevich, M., Hender, T.C., Kwon, O.J., Valovic, M., Medvedev, S., Patel, A., Saarelma, S., Taylor, D., Wilson, H.R. and the MAST Team: 'Ideal MHD stability of the mega-ampere spherical tokamak', Plasma Phys. Control Fusion, 2005, 47, (4), pp. $581-613$

7 Appel, L.C., Akers, R.J., Fullop, T., Martin, R., and Pinfold, T.: 'Observations of CAE's on MAST'. Proc. 31st EPS Conf. on Controlled Fusion and Plasma Physics, 2004, P4.195

8 Appel, L.C., and Hole, M.J.: 'Calibration of the high-frequency magnetic fluctuation diagnostic in plasma devices', accepted, Rev. Sci. Instrum., 2005
9 Heeter, R.F., Fasoli, A.F., Ali-Arshad, S., and Moret, J.M.: 'Fast magnetic fluctuation diagnostics for Alfvén eigenmode and magnetohydrodynamic studies at the Joint European Torus', Rev. Sci. Instrum., 2000, 71, (11), pp. 4092-4106

10 Wheeler, H.A.: 'Simple inductance formulas for radio coils', Proc IRE, 1928, 16, pp. 1398-1400

11 Bartoli, M. Reatti, A and Kazimierczuk, M.K. 'Modelling ironpowder inductors at high frequencies'. Proc. IEEE Industry Applications Soc. Ann. Meeting, 1994, pp. 1225-1232

12 Rhea, R.W.: 'A multimode high-frequency inductor model', Appl Microw. Wirel., 1997, 9, (6), pp. 70-80

13 Horng, T.-S., Wu, J.-M., Yang, L.-W., and Fang, S.-T.: 'A novel modified-T equivalent circuit for modeling LTCC embedded inductors with a large bandwidth', IEEE. Trans. Microw. Theory Tech., 2003, 51, (12), pp. 2327-2333

14 Smythe, W.R.: 'Static and dynamic electricity' (McGraw-Hill, New York, 1950)

15 GoodFellow Catalogue, http://www.goodfellow.com/csp/active/ STATIC/E/Polyamide-imide.HTML, 2004

16 Rhodes and Schwarz website, http://www.rsd.de, 2005

17 Sander, K.F., and Reed, G.A.L.: 'Transmission and propagation of electromagnetic waves' (Cambridge Univeristy Press, 1978)

18 Bartoli, M., Reatti, A., and Kazimierczuk, M.K.: 'High-frequency models of ferrite core inductors', Proc. IEEE Int. Conf. on Industrial Electronics (IECON), 1994, pp. 1670-1675

19 Lagarias, J.C., Reeds, J.A Wright, M.H and Wright, P.E.: 'Convergence properties of the Nelder-Mead simplex method in low dimensions', SIAM J. Optim., 1998, 9, (1), pp. 112-147

20 Simpson, T.L.: 'Effect of a conducting shield on an air-core solenoid', IEEE Trans. Magn., 1999, 35, (1), pp. 508-515 